\title{
Giant vesicles in electric fields $\dagger$
}

\author{
Rumiana Dimova, ${ }^{*}$ Karin A. Riske, $\$$ Said Aranda, Natalya Bezlyepkina, Roland L. Knorr and \\ Reinhard Lipowsky
}

Received 9th March 2007, Accepted 17th April 2007

First published as an Advance Article on the web 4th May 2007

DOI: $10.1039 / \mathrm{b} 703580 \mathrm{~b}$

This review is dedicated to electric field effects on giant unilamellar vesicles, a cell-size membrane system. We summarize various types of behavior observed when vesicles are subjected either to weak AC fields at various frequency, or to strong DC pulses. Different processes such as electrodeformation, -poration and -fusion of giant vesicles are considered. We describe some recent developments, which allowed us to detect the dynamics of the vesicle response with a resolution below milliseconds for all of these processes. Novel aspects on electric field effects on vesicles in the gel phase are introduced.

Max Planck Institute of Colloids and Interfaces, Science Park Golm, 14424 Potsdam, Germany. E-mail: Rumiana.Dimova@mpikg.mpg.de $\uparrow$ The HTML version of this article has been enhanced with colour images.

* Present address: Depto. Biofísica, Unversidade Federal de São Paulo, São Paulo, Brazil

\section{Introduction}

The exposure of biological membranes to electric fields can lead to a variety of responses. The interest towards understanding these responses is motivated by both applications and mechanistic aspects. ${ }^{1-3}$ Studies of cell deformation in alternating electric (AC) fields have been carried out by many groups and effects on the cell shape and motility have been

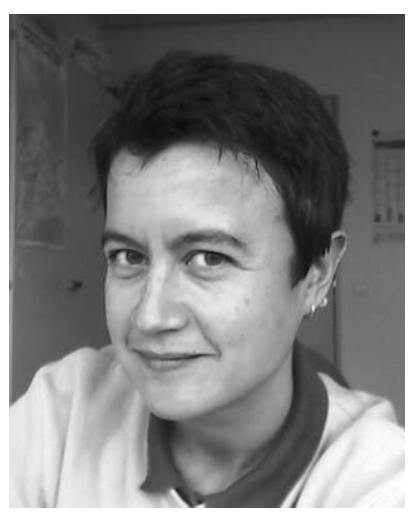

Rumiana Dimova
Rumiana Dimova studied chemical physics and theoretical chemistry at Sofia University (Bulgaria). In 1999, she obtained her PhD at Bordeaux University (France). Since 2000, she is a group leader at the Max Planck Institute of Colloids and Interfaces. Her research interests are in the field of biophysics of model membranes and their interactions.

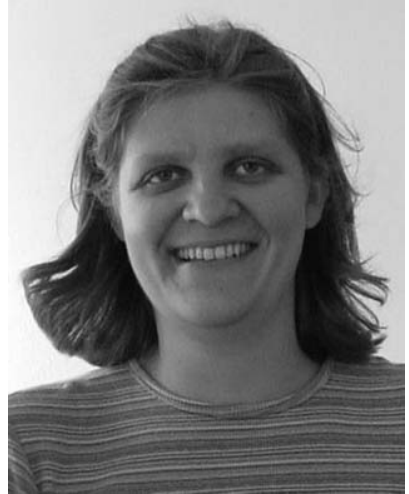

Karin A. Riske
Karin A. Riske obtained her $P h D$ in physics in 2001 at the University of Sao Paulo (Brazil). She was a postdoctoral fellow at the Max Planck Institute of Colloids and Interfaces, and is now a professor at the Federal University of Sao Paulo. Her research interests are lipid bilayers and lipid-biomolecule interactions.

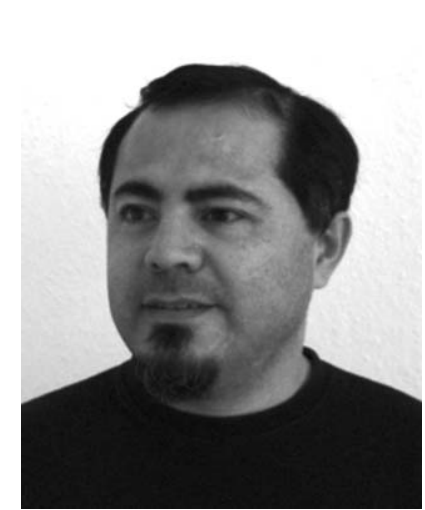

Said Aranda
Said Aranda studied physics in the University of Zacatecas (Mexico). He obtained his master degree in physics at the Research Center for Advanced Studies in Mexico City. Currently he is completing his PhD studies on morphological transitions of vesicles in $A C$ fields in the group of Rumiana Dimova.

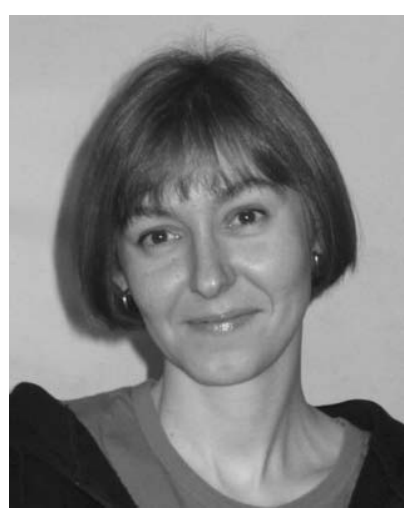

Natalya Bezlyepkina
Natalya Bezlyepkina studied biophysics at the Kharkov State University (Ukraine), and did her master thesis on $D N A$ interaction with metal ions. Now she is a PhD student in the group of Rumiana Dimova, where she is working on domain formation in model membranes. 
reported. $^{4-6}$ Strong electric fields (DC pulses) can cause a significant increase in the electric conductivity and permeability of the cell-plasma membrane. This phenomenon, also referred to as electroporation or electropermeabilization, can be used for introducing various molecules, to which the cell membrane is otherwise impermeable. Due to its efficiency, this method is rapidly becoming an established approach for treatment of carcinoma and melanoma cancer, ${ }^{7-9}$ but it also holds great promise for gene therapy. ${ }^{10,11}$

Giant vesicles provide biomembrane models suitable for systematic measurements of the interaction between electric fields and lipid bilayers. The membrane response to various external factors can be directly visualized under the microscope. ${ }^{12}$ AC fields induce stationary deformation of the vesicles, while the response to short DC pulses is very dynamic and difficult to resolve with standard video recording. Recently, using a fast-imaging digital camera, we were able to capture the immediate response of giant lipid vesicles to electric fields. ${ }^{13-16}$ The vesicle response and relaxation dynamics were recorded with a high temporal resolution using phase-contrast microscopy.

In this review, we summarize our observations on electrodeformation, -poration and -fusion of giant vesicles. We first introduce some basic relations describing the interaction between electric fields and membranes. Then, we consider the shape transitions observed when vesicles are subjected to AC fields for different frequencies and/or media conductivities. The response of vesicles to DC pulses is considered in terms of vesicle deformation and poration. Both, membranes in the fluid and in the gel state, are discussed. At the end, observations on vesicle electrofusion are presented and the application of vesicle electrofusion for creating multidomain membranes is introduced.

\section{Membranes in electric fields: state of the art}

Lipid membranes are essentially impermeable to ions. Thus, in the presence of an electric field, charges accumulate on both sides of the bilayer. For a spherical vesicle of radius $R$ and tilt angle $\theta$ between the electric field and the surface normal, the charge accumulation gives rise to the transmembrane potential ${ }^{17}$

$$
V_{\mathrm{m}}=1.5 R|\cos \theta| E\left[1-\exp \left(-t / \tau_{\text {charg }}\right)\right]
$$

as a function of time $t$. Here, $E$ is the amplitude of the applied electric field and $\tau_{\text {charg }}$ is the membrane charging-time given by ${ }^{17}$

$$
\tau_{\text {charg }}=R C_{\mathrm{m}}\left[1 / \lambda_{\mathrm{in}}+1 /\left(2 \lambda_{\mathrm{ex}}\right)\right]
$$

where $C_{\mathrm{m}}$ is the membrane capacitance, of the order of $1 \mu \mathrm{F} \mathrm{cm}{ }^{-2}$ for fluid lipid membranes, ${ }^{18-20}$ and $\lambda_{\text {in }}$ and $\lambda_{\text {ex }}$ are the conductivities of the internal and external vesicle solution, respectively. For the limiting case of salt-free solutions (the conductivities are a few $\mu \mathrm{S} \mathrm{cm}^{-1}$ ) and for a typical vesicle radius $R=15 \mu \mathrm{m}$, the charging time is $\tau_{\text {charg }} \sim 415 \mu \mathrm{s}$. When the inner and/or outer conductivities are higher, the charging time is shorter. For example, the presence of as little as $5 \mathrm{mM}$ salt in the solution shortens the charging time by about 50 times. For the same vesicle size but at physiological conditions ( $\sim 200 \mathrm{mM}$ salt concentration), $\tau_{\text {charge }} \sim 0.5 \mu \mathrm{s}$.

Eqn (1) and (2) are valid only for a nonconductive membrane. Above some electroporation threshold, the membrane porates, thus becoming conductive and permeable. Beyond this threshold, the transmembrane potential $V_{\mathrm{m}}$ cannot be further increased, and can even decrease due to transport of ions across the membrane. ${ }^{17,21}$

The electroporation phenomenon can also be understood in terms of a stress in the bilayer created by the electric field. The transmembrane potential, $V_{\mathrm{m}}$, induces an effective electrical tension $\sigma_{\mathrm{el}}$, as defined by the Maxwell stress tensor. ${ }^{13,18,22}$ This tension is given by

$$
\sigma_{\mathrm{el}}=\sqrt{\varepsilon \varepsilon_{0}\left[h /\left(2 h_{\mathrm{e}}^{2}\right)\right] V_{\mathrm{m}}^{2},}
$$

where $\varepsilon$ is the dielectric constant of the aqueous solution, $\varepsilon_{0}$ the vacuum permittivity, $h$ is the total bilayer thickness, $(\sim 4 \mathrm{~nm})$, and $h_{\mathrm{e}}$ the dielectric thickness $\left(\sim 2.8 \mathrm{~nm}\right.$ for lecithin bilayers $\left.{ }^{23}\right)$. For vesicles with some initial tension $\sigma_{0}$, the total tension reached during the pulse is

$$
\sigma=\sigma_{0}+\sigma_{\mathrm{el}}
$$

The total membrane tension cannot exceed the tension of rupture. For lipid membranes, the tension of rupture is in the range 5-10 dyn $\mathrm{cm}^{-1}$ also known as lysis tension, $\sigma_{\text {lys }}$, see e.g. ref. 18 and 24. For membranes made of diblock copolymers,

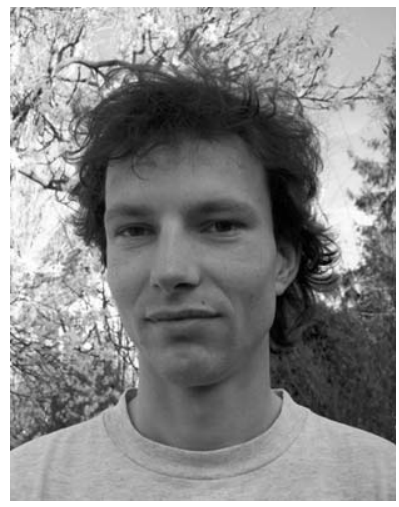

Roland L. Knorr studied nutritional biology at the University of Jena (Germany) and did his master thesis at the Department of Pharmaceutical Biology. He started working on small vesicles and nucleic acid encapsulation at Novosom AG. Currently, he is doing his PhD on model lipid membranes in the group of Rumiana Dimova.

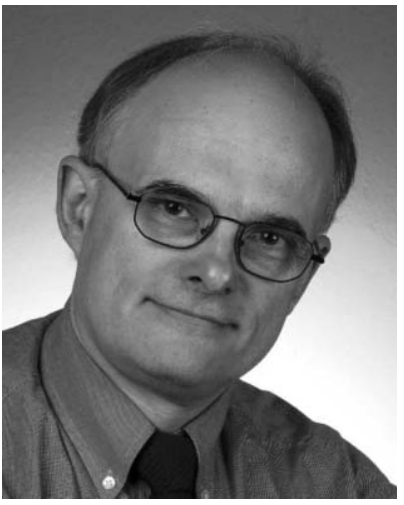

Reinhard Lipowsky obtained his PhD in 1982 at the University of Munich. In 1990, he was appointed full professor at the University of Cologne and director at the Forschungszentrum Jülich. Since 1993, he is a director at the Max Planck Institute of Colloids and Interfaces. He works on fundamental aspects of biomimetic and biological systems. 
the lysis tension is higher and typically exceeds 20 dyn $\mathrm{cm}^{-1} \cdot 25,26$ The lysis tension can be reached either by applying an overall mechanical tension to the vesicle, for example using micropipettes or osmotic pressure, and/or by locally building-up an electric tension. Thus, electroporation occurs when the membrane tension reaches the lysis tension. This corresponds to building-up a certain critical transmembrane potential, $V_{\mathrm{m}}=V_{\mathrm{c}}$. According to eqn (3) and (4), this poration potential $V_{\mathrm{c}}$ depends on the initial membrane tension $\sigma_{0}$, as previously reported. ${ }^{13,18,27}$ The critical transmembrane potential for cell membranes is $V_{\mathrm{c}} \approx 1 \mathrm{~V}$, see e.g. ref. 28 and 29. Similarly, for tension-free vesicles, the critical potential $V_{\mathrm{c}}$ $\sim 1.1 \mathrm{~V}$, (this value was measured for 1-stearoyl-2-oleoyl phosphatidylcholine and dioleoyl phosphatidylglycerol). ${ }^{18}$ The value of the critical poration potential decreases when the initial membrane tension increases. Thus, for a particular vesicle, the electroporation threshold $V_{\mathrm{c}}$ depends on the initial tension of this vesicle. In agreement with this expectation, nonfluctuating vesicles that exhibit an appreciable tension, readily porate at $V_{\mathrm{c}}<1 \mathrm{~V}$, i.e. the fields are weaker than those needed to porate deflated vesicles (with visibly undulating membranes).

Vesicle electroporation is usually induced by applying strong electric fields for a short time interval. Another experimental approach for membrane electroporation involves the usage of much weaker but alternating electric fields. Among experimentalists, working with AC fields is sometimes referred to as "working in the frequency domain" while the usage of DC pulses corresponds to "working in the time domain". Even though AC fields can also be used to induce vesicle poration (see e.g. ref. 30), more studies have focused on vesicle-shape changes induced in the presence of $\mathrm{AC}$ fields. In the next two sections, we discuss the deformation of vesicles when subjected to AC fields and when subjected to DC pulses, respectively. As we will find out, the solution conductivities have a strong influence on the vesicle morphology.

\section{Vesicle deformation in AC fields}

When exposed to AC fields, spherical vesicles assume ellipsoidal shapes. ${ }^{31}$ The type and degree of deformation depends on several parameters, including the field strength, $E$, and frequency, $\omega$, and the conductivities of the media. The tension imposed by the AC field is usually relatively weak $\left(\leqslant 0.1 \mathrm{dyn} \mathrm{cm}^{-1}\right)$ and results mainly in pulling out the membrane undulations. The latter depends on the membrane stiffness. Thus, shape analysis of vesicles deformed in AC fields can be used to measure the bending rigidity of membranes. ${ }^{32,33}$ The tension acting on the membrane can be obtained from: ${ }^{32}$

$$
\sigma_{\mathrm{AC}}=(9 / 8) \varepsilon \varepsilon_{0} E^{2}\left[\left(c_{1}+c_{2}\right)_{\text {pole }}-\left(c_{1}+c_{2}\right)_{\text {eq }}\right]^{-1},
$$

where $\varepsilon$ is the dielectric constant of the aqueous solution as before, and $c_{1}$ and $c_{2}$ are the principal curvatures of the membrane taken either at the equator (indicated by 'eq' as subscript) or at the pole (indicated by 'pole' as subscript). The vesicle curvatures can be estimated from the optical microscopy images of the vesicle, and thus the tension can be calculated. On the other hand, one can measure the relative increase in the vesicle projected area $\delta A / A_{0}$. For low membrane tensions (also known as "the entropic regime"), the latter depends logarithmically on the tension: ${ }^{34,35}$

$$
\delta A / A_{0} \cong\left(k_{\mathrm{B}} T / 8 \pi \kappa\right) \ln \left(\sigma / \sigma_{0}\right)
$$

Here, $A_{0}$ is the initial area for which the tension is $\sigma_{0}$, the membrane tension due to the AC field is $\sigma \approx \sigma_{\mathrm{AC}}, k_{\mathrm{B}}$ is the Boltzmann constant, $T$ is temperature, and $\kappa$ is the bending rigidity of the membrane. Let us note here, that, for a certain field-frequency interval, the tension $\sigma_{\mathrm{AC}}$ acts homogeneously over the whole vesicle, while the electric tension expressed in eqn (3) is local and varies along the vesicle surface and with time (note the angular and temporal dependence of $V_{\mathrm{m}}$ in eqn (1)). From measurements on the vesicle-shape deformation as a function of the field strength (i.e. at different membrane tensions), one can deduce the bending stiffness of the membrane. ${ }^{32,33}$

The shape deformation of vesicles in AC fields has been previously studied, but the dependence of the vesicle morphology on both field frequency and media conductivity has not been determined so far. At low field frequencies (few $\mathrm{kHz}$ ), vesicles in water deform into prolates with the longer axis oriented along the field direction. ${ }^{33}$ At intermediate frequencies (several $\mathrm{kHz}$ ), again for vesicles in water medium, prolateoblate transitions were observed, ${ }^{36-38}$ as theoretically predicted earlier. $^{39,40}$ This behavior was observed also when the conductivity of the external vesicle solution, $\lambda_{\mathrm{ex}}$ was higher than the internal conductivity $\lambda_{\text {in }}$. Interestingly, when the conductivity of the internal solution is raised so that it exceeds the external conductivity, i.e. at $\lambda_{\text {in }}>\lambda_{\mathrm{ex}}$, the prolate-oblate transition is suppressed. With increasing field frequency, the vesicles undergo only the prolate-to-sphere transition. Two examples for the shape evolution of vesicles at different conductivity conditions are provided in Fig. 1.

It is worth mentioning that non-spherical cells have been observed to orient parallel or perpendicular to the field, ${ }^{41}$ whereas, in a shape-analogous way, vesicles in water solutions deform with their largest cross-section also being parallel or

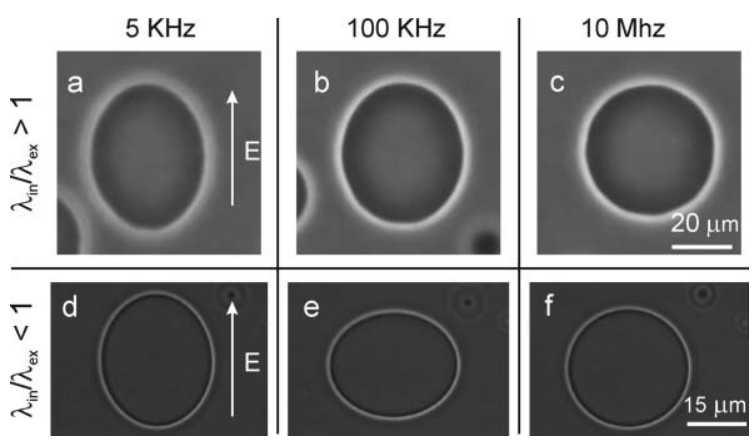

Fig. 1 Two giant vesicles (phase-contrast microscopy) in different conductivity conditions subjected to AC field of $0.2 \mathrm{kV} \mathrm{cm}^{-1}$ and various field frequencies as indicated above the images. The vesicle radii are $21.5 \mu \mathrm{m}$ for the vesicle in $(\mathrm{a}-\mathrm{c}$ ), and $15.2 \mu \mathrm{m}$ for the vesicle in $(\mathrm{d}-\mathrm{f})$. The field direction is indicated with an arrow in (a) and (d). The conductivity conditions are $\lambda_{\text {in }}>\lambda_{\text {ex }}$ for $(\mathrm{a}-\mathrm{c}$ ), where only prolate-tosphere transformation is observed, and $\lambda_{\text {in }}<\lambda_{\text {ex }}$ for (d-f), where prolate-to-oblate and oblate-to-sphere transitions are detected. 


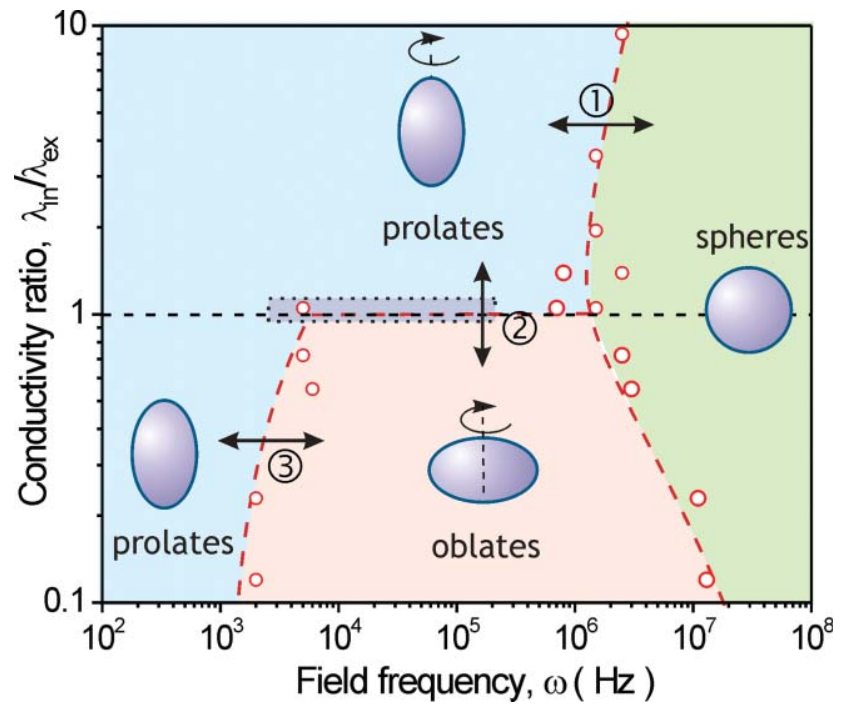

Fig. 2 Morphological diagram of the shape transformations of vesicles in different conductivity conditions and various field frequencies. When the conductivity of the solution inside the vesicles is larger than the one outside, $\lambda_{\text {in }}>\lambda_{\text {ex }}$ (upper part of the diagram), transition from prolate to spherical vesicle (1) is observed with increasing the frequency. For internal conductivities lower than the external one, $\lambda_{\text {in }}<\lambda_{\text {ex }}$ (lower part of the diagram), the vesicle undergoes prolate-to-oblate transition (3) at lower frequencies and oblate-to-sphere transition at higher frequencies. The open circles are experimentally determined. The dashed lines are guides to the eye for the various region boundaries. The area surrounded by the dotted line shows the region previously explored in the literature.

perpendicular to the field. The cell behavior was found to depend on the medium conductivity conditions and field frequency. ${ }^{42,43}$

The frequency dependence of vesicle deformation has been studied in detail, ${ }^{36,39-41,44,45}$ but the influence of solution conductivities has not been well explored. Note that in contrast to cells, one may vary both the external and the internal conductivity for vesicles.

By systematically varying the salt concentrations in the solutions internal and external to the vesicles, as well as the field frequency, we have constructed a morphological diagram of the vesicle-shape transitions occurring at different solutionconductivity ratios $\lambda_{\text {in }} / \lambda_{\text {ex }}$ and various field frequencies $\omega,{ }^{46}$ see Fig. 2. The observations were limited to frequencies above $1 \mathrm{KHz}$ because, around and below this frequency, the field starts to displace the whole vesicle on a micrometre-length scale. The reason for this is charging of the membrane, which induces a net dielecrophoretic force. ${ }^{41}$

Three types of transitions can be distinguished in the morphology diagram (Fig. 2). At high frequency and for arbitrary conductivity condition, the vesicles assume spherical shapes (the transition line is indicated with (1) in Fig. 2), see also Fig. 1c,f. A simple reasoning explaining the spherical morphology is that at this high frequency (several $\mathrm{MHz}$ ) the ions in the solution can no longer follow the field oscillations and thus, the vesicles are no longer deformed. It is easy to see that such frequencies correspond to travel distances of the ions of the order of nanometres, which is not enough to induce detectable deflection of the membrane. This transition is not

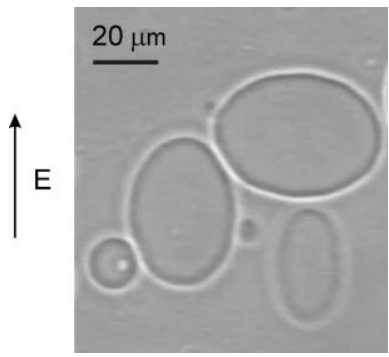

Fig. 3 Coexistence of vesicle shape deformations at the prolateoblate transition line defined by the condition $\lambda_{\text {in }} / \lambda_{\text {ex }} \cong 1$. The field direction is indicated with an arrow. The conductivity conditions for this vesicle solution are such that $\lambda_{\mathrm{in}} / \lambda_{\mathrm{ex}}=1.05$. The field strength is $0.2 \mathrm{kV} \mathrm{cm}^{-1}$ and the field frequency is $\omega=1 \mathrm{MHz}$.

abrupt, but occurs within a frequency interval between $1 \mathrm{MHz}$ and $10 \mathrm{MHz}$.

The second type of transition occurs as one changes the conductivity conditions (the transition line is indicated with (2) in Fig. 2). At intermediate frequencies, between a few $\mathrm{KHz}$ and several $\mathrm{MHz}$, the vesicles assume prolate shapes when the conductivity inside is higher than the external one, see Fig. $1 \mathrm{~b}$. The long axis of the vesicles is oriented parallel to the field. With optical microscopy, only a two-dimensional projection of the vesicle shape can be detected (see Fig. 1). However, the deformation of the vesicles is axially symmetric around the electric field direction. In the opposite case, for $\lambda_{\text {in }} / \lambda_{\text {ex }}<1$, the vesicles become oblate, see Fig. 1e. At the border conductivity range, $\lambda_{\text {in }} / \lambda_{\text {ex }} \cong 1$, coexistence of the two shapes can be observed, see Fig. 3. Again, a simple interpretation for this type of transition can be given by considering the effect of the ions acting on the membrane. For higher internal conductivities $\left(\lambda_{\text {in }}>\lambda_{\text {ex }}\right.$ ), the pressure inside the vesicle exerted by the ions when the field is on, is higher than the external one, because the ion concentration inside is higher. This leads to a force elongating the vesicle along the direction of the field. In the opposite case, the higher ion concentration in the external media causes compression of the vesicle from the outside along the direction of the field, thus leading to an oblate deformation.

The third type of transition is observed only for the conductivity condition $\lambda_{\text {in }} / \lambda_{\text {ex }}<1$ (indicated with (3) in Fig. 2). At low frequencies (below a few $\mathrm{MHz}$ ) the vesicles are prolate, and at higher frequency the shape changes to oblate (see Fig. 1d,e). This frequency-induced shape transformation has been investigated by several groups. ${ }^{36,38,40,47}$ Mainly vesicles in water solution were studied, where the conductivity condition was such that $\lambda_{\text {in }} / \lambda_{\mathrm{ex}} \cong 1$. The transition has been interpreted as an effect due to anisotropy of the membrane dielectric-permittivity ${ }^{47}$ or a transition of the water media from the conductive regime (low frequencies) to the dielectric regime (high frequencies). ${ }^{36}$ Another interpretation involved finding the steady-state solutions of the dynamical equations determined by the electric field inside and around a vesicle. ${ }^{40}$

\section{Vesicle response to DC pulses}

While the discussion of vesicles exposed to AC fields was limited to stationary shapes, DC pulses induce short-lived 
shape deformations. The pulse duration is usually varied between several microseconds to milliseconds, while studies on cells have investigated a much wider range of pulse durations from tens of nanoseconds to milliseconds and even seconds. ${ }^{1}$ Because the application of both AC fields and DC pulses creates a transmembrane potential, vesicle deformations of similar nature are to be expected in both cases. Indeed the AC field frequency, $\omega$, should be compared to the inverse duration of the DC pulse, $1 / t_{\mathrm{p}}$. This correspondence should be valid for a certain shape of the AC field signal (whether sinusoidal, rectangular or other shape) and of the DC pulse (square, triangular, etc.). For example, applying a square-wave AC field is analogous to applying a sequence of square-wave DC pulses with alternating electrode polarity. At equivalent conductivity conditions, a DC pulse of duration $t_{\mathrm{p}}=100 \mu \mathrm{s}$ should induce a deformation similar to the one obtained for a square-wave AC field of frequency $\omega=5 \mathrm{MHz}$, i.e. a correspondence between $\omega$ and $1 /\left(2 t_{\mathrm{p}}\right)$ is to be Nexpected. However, the working field strength for the DC pulses is usually higher by several orders of magnitude. Thus, the degree of deformation can be different. Note that in theoretical works, very often an angular field frequency, $\omega^{\prime}$, is used, i.e. $\omega^{\prime}=2 \pi \omega$. The latter is preferred because it simplifies the mathematical treatment of the problem.

Vesicle deformation induced by DC pulses has been studied theoretically. ${ }^{48-50}$ The direct experimental visualization of the vesicle response was achieved from observations on giant vesicles. ${ }^{13,14} \mathrm{~A}$ few other experiments have been performed but mainly with small vesicles, which are hundreds of nanometres in size. ${ }^{45,51,52}$ Poration of small vesicles induced by DC pulses has attracted stronger interest. ${ }^{52-55}$ Because of the small size of the vesicles, direct observation of the deformation and poration is not feasible. In addition, membrane tension and curvature may play a significant role. The vesicle response has been detected using turbidity, absorbance, and conductivity measurements where microsecond resolution can be achieved.

Experiments on giant vesicles made of lipids and polymers are of special relevance because their size is comparable to cells and, in addition, it allows for direct observation using optical microscopy. ${ }^{25,56-59}$ However, microscopy observation of effects caused by electric pulses on lipid giant vesicles is difficult because of the short duration of the pulse. A typical video frequency of 30 frames per second would provide a snapshot every 33 milliseconds. Keeping in mind that the pulse duration is about 3 orders of magnitude shorter, the immediate dynamics of the vesicle response would be indiscernible at such acquisition speed. A partial solution to this problem is to use pulsed laser fluorescence microscopy for the visualization of the vesicle response, ${ }^{55}$ but appropriate fluorescent labeling of the membrane is needed. Another approach involves slowing down the processes by using a highly viscous fluid, e.g. glycerin, as the medium instead of water. ${ }^{57}$ However, such an approach can set physically different limitations on the response dynamics of the membrane. In addition, it may lead to a change in the hydration of the lipid bilayer and, correspondingly, alter the membrane properties.

To deal with this time-resolution challenge, recently in our lab, we have used a fast-imaging digital camera to record the giant vesicle response with a high temporal resolution, up to
30000 frames per second (fps), i.e. acquiring 1 image every $33 \mu \mathrm{s} .{ }^{13-16}$ This unprecedented time resolution overcomes the observation limits imposed by video frequency. In this setup no labeling whatsoever is necessary and there is no need for using viscous solvents as working medium. Using the fast digital camera, we were able to resolve the dynamics of the vesicle response during and after the applied DC pulse.

The following sections introduce some characteristic features of the deformation of spherical vesicles when subjected to square-wave pulses. We first consider the vesicle response in the absence of salt. To enhance the microscopy imaging, the external medium of the vesicles was glucose solution. Inside, the vesicles contained isotonic sucrose solution. In this case, the conductivity ratio was $\lambda_{\text {in }} / \lambda_{\mathrm{ex}} \approx 1.3$. The pulse duration was varied between $50 \mu$ s and $300 \mu \mathrm{s}$, thus similar responses as in $\mathrm{AC}$ fields in the frequency region $1.5-10 \mathrm{KHz}$ are to be expected. Indeed, similarly to the behavior in AC fields, as classified in the morphological diagram in Fig. 2, the vesicles subjected to DC pulses assume prolate shapes with the long axis parallel to the field direction. However, the degree of deformation induced by AC fields is much smaller because the fields are much weaker compared to those of the DC pulses.

We also discuss the dynamics of the vesicle relaxation in the absence and presence of pores in salt-free solutions. Then, we consider vesicles in the presence of salt, for which surprising cylindrical deformations are observed. At the end of this section, we introduce some observations on the electroporation of vesicles in the gel phase.

\subsection{Dynamics of vesicle deformation in the absence of salt}

Spherical vesicles subjected to electric pulses assume ellipsoidal shapes, which relax back to the initial vesicle shapes after the end of the pulse. The degree of deformation of an ellipsoidal vesicle can be characterized by the aspect ratio of the two principal radii, $a$ and $b$ (see sketch in Fig. 4). For $a / b=1$ the vesicle is a sphere. The relaxation dynamics of this aspect ratio depends on whether the vesicle has been porated or not. In the absence of poration, the relaxation can be described by a single exponential with a characteristic decay time, $\tau_{1}$. Fig. 4 gives one example of the response of a giant vesicle, which is initially spherical. The pulse conditions in this case build-up a transmembrane potential, which is below the poration limit. The maximum deformation of this vesicle corresponds to about $10 \%$ change in the vesicle aspect ratio. The degree of vesicle deformation depends on the initial tension of the vesicle as well as on the excess area. The latter is defined as an excess compared to the area of a spherical vesicle of the same volume.

The typical decay time for the relaxation of non-porated vesicles, $\tau_{1}$, is of the order of $100 \mu \mathrm{s}$. It is defined by the relaxation of the total membrane tension achieved at the end of the pulse, which is the sum of the electrotension $\sigma_{\mathrm{el}}$ and the initial tension $\sigma_{0}$, see eqn (4). Thus, $\tau_{1}$ relates mainly to the relaxation of membrane stretching, $\tau_{1} \sim \eta_{\mathrm{m}} / \sigma$, where $\eta_{\mathrm{m}}$ is the surface viscosity of the membrane (the surface viscosity of a membrane has units [bulk viscosity] $\times$ [bilayer thickness], i.e. dyn $\mathrm{s} \mathrm{cm}^{-1}$ ). The shear-surface viscosity of the membrane is $\eta_{\mathrm{s}} \approx 3 \times 10^{-6} \mathrm{dyn} \mathrm{s} \mathrm{cm}{ }^{-1}, 60,61$ corresponding to values reported for the diffusion constant of molecular probes in lipid 

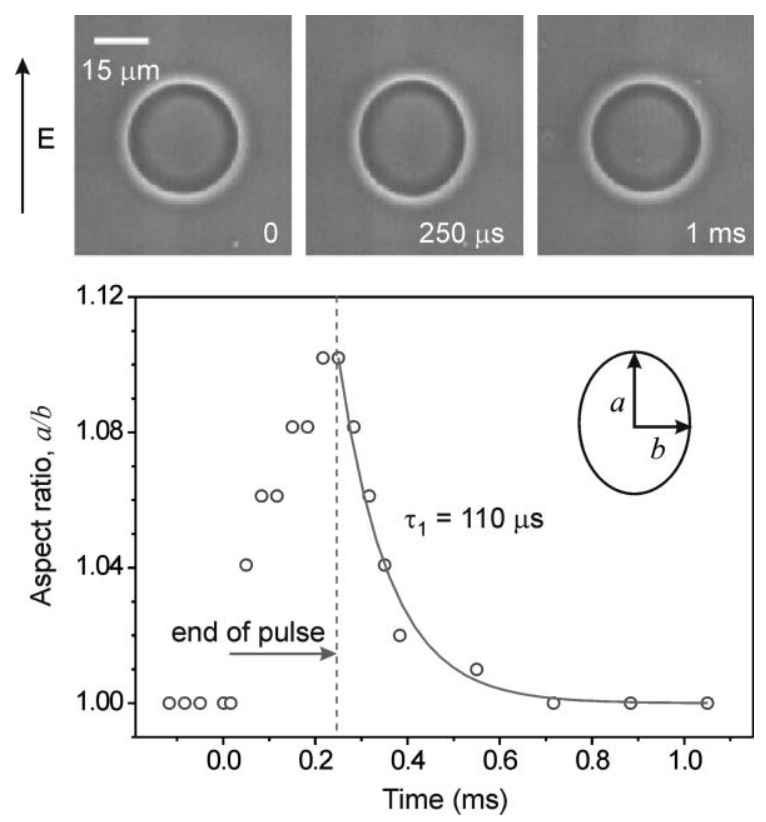

Fig. 4 Response of a vesicle subjected to a square-wave pulse $E=$ $1 \mathrm{kV} \mathrm{cm}^{-1}, t_{\mathrm{p}}=250 \mu \mathrm{s}\left(V_{\mathrm{p}}<V_{\mathrm{c}}\right)$. Three snapshots of the vesicle before and after the pulse are given above (the time is indicated at the lower right corner of each micrograph). The direction of the field is indicated with an arrow. Time $t=0$ was set as the beginning of the pulse. The image acquisition rate was $30000 \mathrm{fps}$. The degree of deformation $(a / b)$ attained by the vesicle is plotted. The dashed line indicates the end of the pulse. The solid line is an exponential fit with a decay time $\tau_{1}$.

bilayers $D \approx 10^{-13}-10^{-12} \mathrm{~m}^{2} \mathrm{~s}^{-1} \cdot{ }^{62}$ Some other measurements of the dilatational-surface viscosity ${ }^{63}$ determined by following the dynamics of macropores on giant vesicles suggest a value, which is two orders of magnitude higher, $\eta_{\mathrm{d}} \approx 3.5 \times$ $10^{-4} \mathrm{dyn} \mathrm{s} \mathrm{cm}^{-1}$. This is probably because the reported experiments are related to dilation rather than to shearing the membrane. Thus, choosing for the membrane viscosity the value of $\eta_{\mathrm{d}}$ given in ref. 63 is more appropriate since it has been measured for membrane poration. Coming back to $\tau_{1}$, for membrane tensions of the order of $5 \mathrm{dyn}^{-1}$ (which should be around the maximum tension before the membrane ruptures) one obtains $\tau_{1} \sim \eta_{\mathrm{d}} / \sigma \sim 100 \mu \mathrm{s}$, which corresponds to the experimentally measured value.

\subsection{Electroporation dynamics}

Electroporation is often used to introduce molecules like proteins, foreign genes (plasmids), antibodies, drugs, etc. into cells. Several studies in this area have already been conducted in an attempt to optimize and model the influence of the parameters, like the intensity and duration of the electric field. ${ }^{64-69}$ The pores formed during electroporation can reach various sizes depending on the location on the vesicle or cell surface, see e.g. ref. 29 and 70 and work cited therein. For the case of plane-parallel electrodes, the poration occurs predominantly in the area at the poles of the vesicle facing the electrodes. This is because the transmembrane potential attains its maximal values at the two poles as expressed by the angular dependence in eqn (1). With optical microscopy, only pores that are of diameter larger than about half a micron can be resolved. We refer to them as macropores since they are much larger than the average ones. With fast camera observation on giant vesicles, macropores can be visualized in the following way. If the vesicles are prepared in sucrose solution and then diluted in isotonic glucose solution, the refractive indices of the internal and external vesicle media are different. Thus, vesicles appear as dark objects on a light gray background, see e.g. the snapshots in Fig. 1. When macropores are formed, there is an efflux of the darker sucrose solution. Fig. 5 shows an example of an electroporated vesicle.

The lifetime of macropores, $\tau_{\text {pore }}$, observed in vesicles in the fluid state, varies with pore diameter, $d_{\text {pore }},{ }^{57}$ and depends on the membrane-edge tension, $\gamma$, and the membrane dilatationalviscosity, $\tau_{\text {pore }} \sim d_{\text {pore }} \eta_{\mathrm{d}} / \gamma .{ }^{13}$ For vesicles with low tension, the lifetime $\tau_{\text {pore }}$ is typically shorter than $30 \mathrm{~ms}$, see ref. 13 .

The relaxation dynamics of porated vesicles is significantly different from the one of non-porated vesicles. ${ }^{13}$ Indeed, two different types of dynamics can be distinguished for porated vesicles. The relaxation of vesicles with no excess area is described by a single exponential decay, while vesicles with excess area exhibit two characteristic decay times. These two cases for vesicles above the poration limit are illustrated in Fig. 6. The maximum deformation achieved in both cases is much higher than the one observed for non-porated vesicles (compare the maximum values of $a / b$ in Fig. 4 and in Fig. 6). Naturally, vesicles with excess area deform much more than those without (compare the two curves in Fig. 6). The relaxation of porated vesicles completes over a much longer time than that of nonporated ones (compare the values on the abscissa in Fig. 4 and in Fig. 6; note that the abscissa in Fig. 6 is in logarithmic scale and the one in Fig. 4 is in linear scale). For vesicles with no excess area, the relaxation time is $\tau_{2} \cong 7 \pm 3 \mathrm{~ms}$. When the vesicles have some excess area, the relaxation proceeds in two steps, fast relaxation characterized by $\tau_{2}$, and a second, longer, relaxation with decay time, $\tau_{3} ; 0.5 \mathrm{~s}<\tau_{3}<3 \mathrm{~s}$.
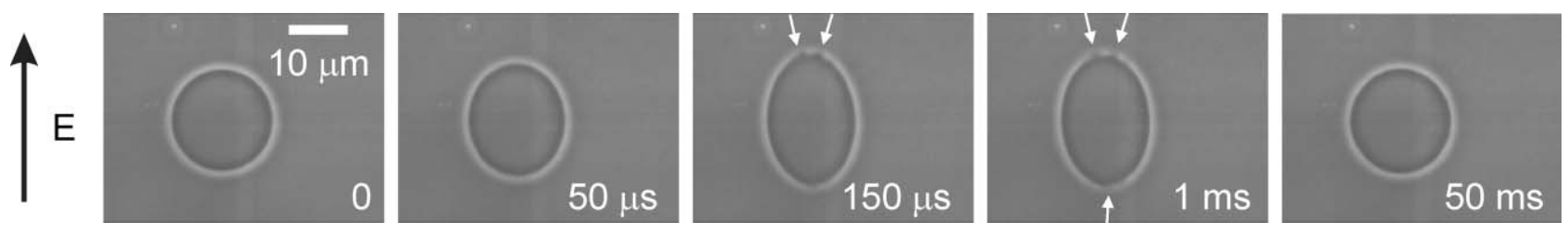

Fig. 5 Poration of a vesicle (phase-contrast microscopy; the internal solution is sucrose and the external one is glucose; acquisition rate $20000 \mathrm{fps}$ ). The DC pulse duration is $200 \mu$ s and the field strength is $1.4 \mathrm{kV} \mathrm{cm}^{-1}$. The direction of the field is indicated by the arrow on the left. The time period in the lower right corner of each snapshot is the time after the beginning of the pulse. The arrows in the second and third snapshots indicate pores at the vesicle poles visualized by dark eruptions of sucrose solution leaking out of the vesicle. The pores reach a size up to $4 \mu \mathrm{m}$ in diameter. 


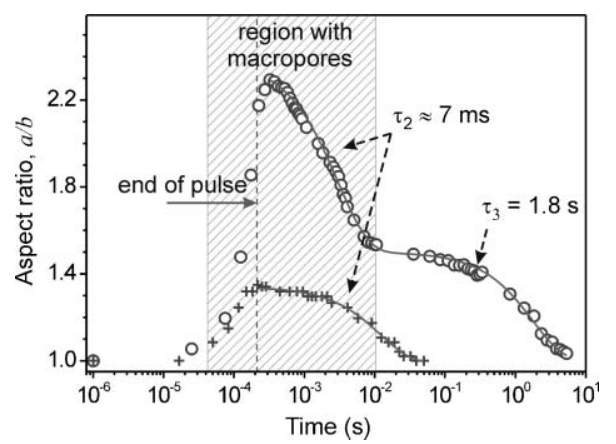

Fig. 6 Data from the response and relaxation of two vesicles, in which macropores were observed. One of the vesicles (crosses) did not have excess area and the relaxation is described by a single exponential fit (solid curve) with a decay time $\tau_{2}$. The other vesicle (open circles) had excess area and its relaxation is described by a double exponential fit (solid curve) with decay times $\tau_{2}$ and $\tau_{3}$ as described in the text. In both cases, the pulse strength was $E=$ $2 \mathrm{kV} \mathrm{cm}^{-1}$ and the pulse duration was $t_{\mathrm{p}}=200 \mu \mathrm{s}$ (the end of the pulse is indicated with a vertical dashed line). The time $t=0$ was set as the beginning of the pulse. The shaded area indicates the time interval when macropores were optically detected. The radii of the vesicles were around $10 \mu \mathrm{m}$.

The relaxation process associated with $\tau_{2}$, takes place during the time interval when pores are present (see shaded region in Fig. 6). Thus, $\tau_{2}$ is determined by the closing of the pores, $\tau_{2} \sim$ $\eta_{\mathrm{d}} r_{\text {pore }} /(2 \gamma)$. Here, $r_{\text {pore }}$ is the pore radius and $\gamma$ is the line energy per unit length. The latter is $\gamma \sim(\kappa / 2) h$, where $\kappa$ is the bending stiffness of the bilayer and $h$ is the membrane thickness $\sim 4 \mathrm{~nm}$, thus yielding for $\gamma$ a value of the order of $10^{-6}$ dyn $\left(10^{-11} \mathrm{~J} \mathrm{~m}^{-1}\right) \cdot{ }^{30}$ For a typical pore radius of $1 \mu \mathrm{m}$ one obtains $\tau_{2} \sim 10 \mathrm{~ms}$.

The relaxation time $\tau_{3}$ is related to the presence of some excess area available for shape changes. The latter can be characterized by a dimensionless volume-to-area ratio $v=(3 V / 4 \pi)(4 \pi / A)^{3 / 2}$, where $V$ is the vesicle volume, $V=$ $(4 \pi / 3) a b^{2}$. This reduced volume $v$ is 1 when the vesicle is a sphere and smaller than 1 in the rest of the cases. The relaxation described by $\tau_{3}$ is associated with the process of pushing-away the volume of fluid involved in the ellipsoidal vesicle deformation compared to a relaxed spherical shape. The restoring force is related to the bending elasticity of the lipid bilayer. Then, the decay time can be presented as $\tau_{3} \sim\left(4 \pi \eta R^{3} / 3 \kappa\right)(1 / v-1)$, where $\eta$ is the bulk viscosity of sucrose/glucose solution, as above, and $\kappa$ is the bending elasticity modulus of the membrane. For egg-PC $\kappa \approx$ $10^{-12}$ erg. $^{71,72}$ Thus, for typical values of $v$ between 0.99

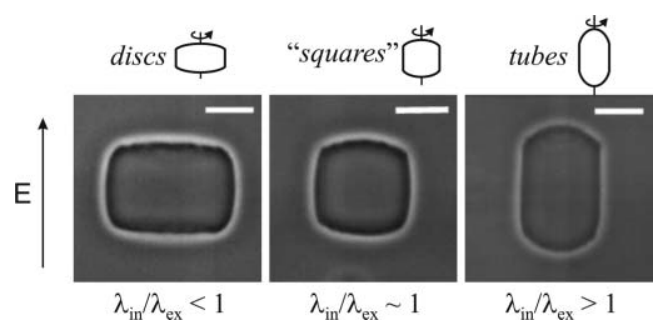

Fig. 7 Deformation of vesicles at different conductivity conditions subjected to DC pulses. Schematic illustrations of the cross-sections of the vesicles are given above every snapshot. The field direction is indicated with an arrow on the left. The presence of salt in the vesicle exterior causes flattening of the vesicle membrane into disc-like, "square"-like, and tube-like shapes, whereby the overall vesicle shape depends on the conductivity ratio. The scale bars correspond to $15 \mu \mathrm{m}$.

and 0.94 , one obtains $\tau_{3}$ between approximately 0.5 and $3 \mathrm{~s}$, which corresponds excellently to the measured data. All characteristic decay times for vesicle relaxation and the macropore lifetime are summarized in Table 1.

\subsection{Cylindrical deformations of vesicles}

In the presence of salt in the vesicle exterior (e.g. $\mathrm{NaCl}$ solution with concentration above $0.1 \mathrm{mM}$ ), unusual shape changes are observed. ${ }^{14}$ The vesicles assume cylindrical shapes during the pulse. These deformations are short-lived (their lifetime is about $1 \mathrm{~ms}$ ) and occur only in the presence of salt outside the vesicles, irrespective of their inner content. When the solution conductivities inside and outside are the same, $\lambda_{\text {in }}=\lambda_{\mathrm{ex}}$, vesicles with square cross-section are observed. For the case where $\lambda_{\text {in }}<\lambda_{\text {ex }}$, the vesicles assume disc-like shapes, while in the opposite case, $\lambda_{\text {in }}>\lambda_{\text {ex }}$, they deform into long cylinders with rounded caps, see Fig. 7. The transition from tubes to discs is analogous to transition (2) (from prolates to oblates) in Fig. 2 observed in AC fields.

The detected cylindrical deformations are non-equilibrium shapes and have a very short lifetime, which is why they have not been observed for standard video acquisition speed. The flattening of the vesicle walls starts during the applied pulse and is observed throughout a period of about one millisecond. The formation of these shapes is not well understood. One possible hypothesis is related to electrophoretic forces exerted by the ions in the external solution, for details see ref. 14 . Another interpretation might be an electrohydrodynamic instability caused by electric fields interacting with flat membranes, which was predicted to increase the membrane roughness. ${ }^{73}$

Table 1 Characteristic times of vesicle relaxation and poration

\begin{tabular}{llll}
\hline Decay times & Specification & Typical values & Dependence on membrane material properties ${ }^{a}$ \\
\hline$\tau_{1}$ & Decay time for conditions of no poration & $\sim 100 \mu \mathrm{s}$ & $\tau_{1} \sim \eta_{\mathrm{d}} / \sigma$ \\
$\tau_{2}$ & Decay time for conditions of poration & $3-10 \mathrm{~ms}$ & $\tau_{2} \sim \eta_{\mathrm{d}} r_{\text {pore }} /(2 \gamma)$ \\
$\tau_{3}$ & Relaxation time of vesicles with excess area & $0.5-3 \mathrm{~s}$ & $\tau_{3} \sim\left(4 \pi \eta R^{3} / 3 \kappa\right)(1 / v-1)$ \\
$\tau_{\text {pore }}$ & Lifetime of macropores & $<30 \mathrm{~ms}$ & $\tau_{\mathrm{pore}} \sim d_{\text {pore }} \eta_{\mathrm{d}} / \gamma$ \\
${ }^{a}$ In the expressions here, $\eta_{\mathrm{d}}$ is the membrane dilational viscosity, $\sigma$ is the total membrane tension, $r_{\text {pore }}$ is the pore radius, $\gamma$ is the line energy \\
per unit length, $\eta$ is the bulk viscosity of the media, $\kappa$ is the bending elasticity modulus of the membrane, $R$ is the vesicle radius, $v$ is the \\
reduced volume, and $d_{\text {pore }}$ is the pore diameter.
\end{tabular}



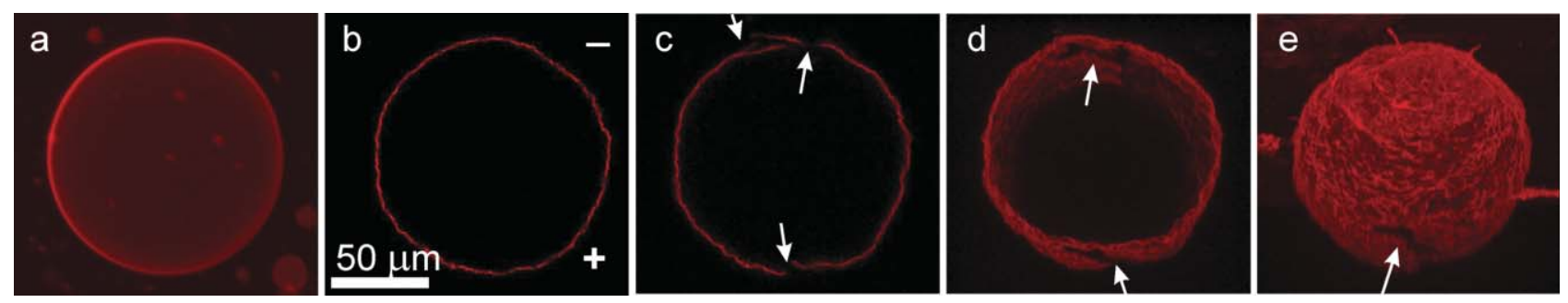

Fig. 8 Electroporation of a fluorescently-labeled DPPC vesicle in the gel phase as imaged with confocal microscopy. (a) A 3-D projection averaged image of a vesicle in the fluid phase. (b-e) Images of a vesicle in the gel phase: equatorial sections of the vesicle before (b) and after poration (c) caused by an electric pulse of $6 \mathrm{kV} \mathrm{cm}^{-1}$ and duration $300 \mu$ s. The electrode polarity is indicated with plus (+) and minus $(-)$ signs in (b). The arrows in (c) show the ruptured zones at the vesicle poles. A stripe from the equatorial area of the vesicle which is 30 micrometres wide and slightly rotated around the horizontal axis, shows the ruptured places in the membrane at the north and south poles (d) as indicated with arrows. A complete 3-D projection average image of the same vesicle (again rotated around the $x$-axis) shows better the crack on the southern pole of the vesicle (e) pointed by the arrow. Contrary to vesicles in the fluid phase (a), pores formed on vesicles in the gel phase (e) do not reseal over a period of at least ten minutes.

\subsection{Electrodeformation and -poration of membranes in the gel phase}

When lipid bilayers undergo a fluid-to-gel transition, the shear-surface viscosity and the bending stiffness of the membrane increase drastically. ${ }^{61}$ Thus, it is to be expected that the dynamics of electrodeformation and poration of vesicles in the gel and in the fluid phase would differ significantly. Presumably, the lifetime of pores formed on vesicles in the gel phase would be much longer. We attempted to visualize such pores using confocal microscopy on giant vesicles porated while being in the gel phase. The vesicles were prepared from dipalmitoyl phosphatidylcholine (DPPC). At room temperature, this lipid is in the gel phase. The main phase-transition temperature of DPPC is $41.5{ }^{\circ} \mathrm{C} .{ }^{74}$ Electroporation studies on large unilamellar DPPC vesicles (about $100 \mathrm{~nm}$ in diameter) in the gel phase suggested the formation of transient pores. ${ }^{53}$ These pores were presumably much smaller than $100 \mathrm{~nm}$, and the poration was reported not to induce a global damage of the vesicles. In contrast, the pores observed here for giant unilamellar vesicles induced by strong DC pulses are very stable, they are several micrometres in size, and resemble cracks on a rough solid shell, see Fig. 8. It was believed that the electroporation of small vesicles in the gel state is a reversible process. ${ }^{53}$ However, our observations suggest that the formation of cracks on giant vesicles in the gel phase appear to be an irreversible process on a laboratory timescale. Within a period of more than ten minutes, we did not observe resealing of these cracks. In some cases, the vesicles collapsed at a later stage.

Compared to the macropores in fluid membranes, which have a lifetime shorter than about $30 \mathrm{~ms}$, the pore resealing in vesicles in the gel state seems to be arrested. This is to be expected, considering the dependence of the pore lifetime, $\tau_{\text {pore, }}$ on the membrane viscosity, see Table 1 . The shearsurface viscosity diverges when the membrane crosses the fluid-to-gel transition ${ }^{61}$ and thus the resealing process for pores formed on membranes in the gel phase is suppressed.

An additional difference from the behavior of membranes in the fluid phase is the poration condition for membranes in the gel phase. The latter is characterized by the critical transmembrane potential $V_{\mathrm{c}}$. For cell membranes and tension-free lipid bilayers in the fluid phase, $V_{\mathrm{c}} \sim 1 \mathrm{~V}^{18,28,29}$ (note that large unilamellar vesicles electroporate at lower field strength because they are usually under tension). We attempted to estimate the critical transmembrane potential for DPPC in the gel phase in the following way. Giant vesicles were subjected to DC pulses of fixed duration and increasing field strength. The vesicles were inspected for pores before and after the applied pulse. From the field strength at which pores were detected, we calculated the critical poration potential using eqn (1) and (2) (for the time $t$ in eqn (1), we take the pulse duration which was kept constant). To estimate the membrane capacitance per surface area, $C_{\mathrm{m}}$, of the bilayer, we used the expression ${ }^{41}$

$$
C_{\mathrm{m}}=\varepsilon_{\mathrm{m}} \varepsilon_{0} / h,
$$

where $\varepsilon_{\mathrm{m}}$ is the dielectric permittivity of the membrane, $\varepsilon_{\mathrm{m}} \approx$ $5,{ }^{75}$ and $h$ is the membrane thickness as before. The parameter that can differ depending on the state of the membrane, fluid or gel, is the membrane thickness $h$. For example, for DPPC in the fluid state $h \approx 4.7 \mathrm{~nm}$, while in the gel phase $h \approx 5.2 \mathrm{~nm}$. $^{76}$ This corresponds to an overall decrease of the membrane capacitance of bilayers that undergo the fluid-to-gel transition. This decrease was experimentally confirmed to be between 10 and $15 \%$, corresponding well to the change in the membrane thickness. ${ }^{77}$ Thus, the characteristic charging time for membranes in the gel phase as determined from eqn (2) should be shorter. Following this approach, the estimated critical transmembrane potential for poration of membranes in the gel state is $V_{\mathrm{c}}=1.5 \pm 0.2 \mathrm{~V}$. This value is higher than $V_{\mathrm{c}}$ measured for fluid membranes. This implies that the membrane in the gel state is more difficult to porate, presumably indicating a higher lysis tension.

\section{Vesicle electrofusion}

When a DC pulse is applied to a couple of vesicles in the fluid state, which are in contact and oriented in the direction of the field, electrofusion can be observed. The application of the AC field to a vesicle suspension leads to their orientation and alignment of the vesicles in the field, forming pearl chains. This phenomenon is also observed with cells ${ }^{3,78}$ and is due to dielectric screening of the field. When the suspension is dilute, 


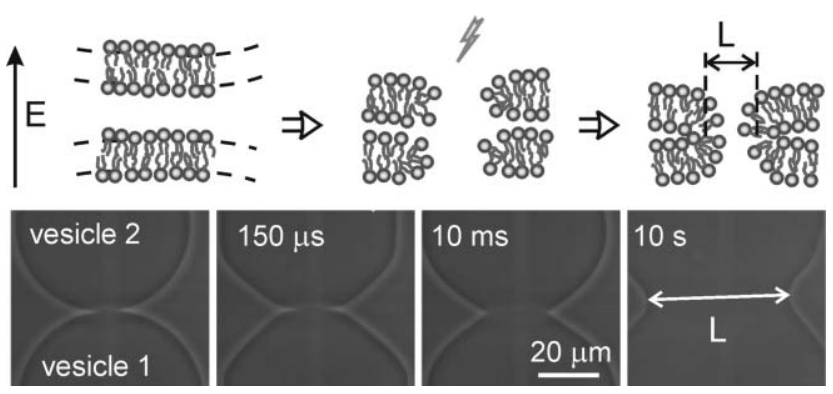

Fig. 9 (upper panel) Schematic illustration of the possible steps of the electrofusion process: two lipid vesicles are brought into contact (only the membranes in the contact zone of the vesicles are sketched), followed by electroporation and formation of a fusion neck of diameter $L$. (lower panel) Micrographs from electrofusion of a vesicle couple. Only segments of the vesicles are visible. The external solution contains $1 \mathrm{mM} \mathrm{NaCl}$, which causes flattening of the vesicle walls (see second snapshot). The amplitude of the DC pulse was $2.4 \mathrm{kV} \mathrm{cm}{ }^{-1}$, and its duration was $120 \mu \mathrm{s}$. The starting time $t=0$ corresponds to the beginning of the DC pulse. The image acquisition rate was $20000 \mathrm{fps}$.

two vesicles can be brought together via the $\mathrm{AC}$ field and aligned. A subsequent application of a DC pulse to such a vesicle couple can lead to fusion. The necessary condition is that poration is induced in the contact area between the two vesicles. The possible steps of the electrofusion of two membranes are schematically illustrated in Fig. 9.

Membrane fusion is a fast process. The time needed for the formation of a fusion neck can be rather short as follows from electrophysiological methods applied to the fusion of small vesicles with cell membranes. ${ }^{79-81}$ The time evolution of the observed membrane capacitance indicates that the formation of the fusion neck is presumably faster than $100 \mu$ s.

Previously, the limits of direct observation with optical microscopy were in the range of milliseconds. The usage of fast digital imaging, shifted the resolution limit by about two orders of magnitude and allowed studying of the fusion dynamics with microsecond resolution. ${ }^{15,16}$ An example of a few snapshots taken from the electrofusion of two vesicles in the presence of salt is given in Fig. 9. Note that in this case, the overall deformation of each vesicle corresponds to the cylindrical shapes as observed with individual vesicles in the presence of salt (see section 4.3).

From such micrographs, one can measure the fusion-neck diameter, denoted by $L$ in Fig. 9, and follow the dynamics of its expansion. In Fig. 10, the time evolution of the fusion neck of one vesicle couple is given. The data spans more than 5 orders of magnitude in time. A first inspection of Fig. 10 shows that two stages of the fusion process can be distinguished, first a very fast one, followed by a slower one (see the inset in Fig. 10, where the same data is plotted in linear timescale).

The initial stage of the fusion process is surprisingly fast. Detailed image analysis of the fusion zone demonstrates that the opening of the fusion neck with a radius larger than $\sim 10 \mu \mathrm{m}$ takes place within several frames after the applied pulse, e.g. within approximately $500 \mu$ s, see the early time data points in Fig. 10. This implies an average expansion velocity of about $2 \mathrm{~cm} \mathrm{~s}^{-1}$. Intuitively, one would relate this to fast relaxation of the membrane tension, whereby the dissipation

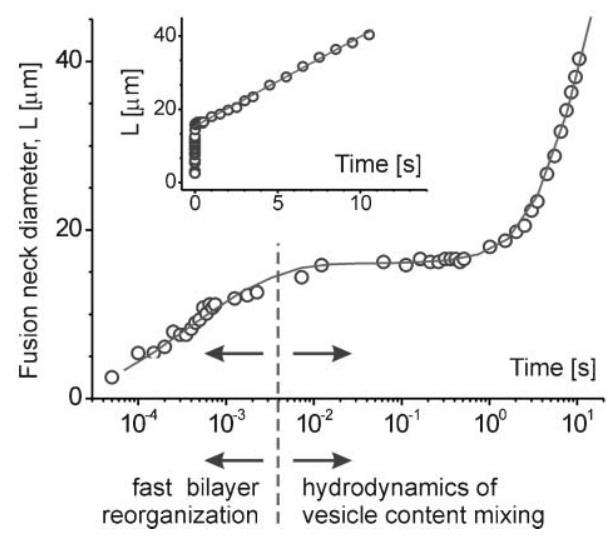

Fig. 10 Time evolution of the fusion neck, $L$ formed between two vesicles about $15 \mu \mathrm{m}$ in radius. The solid curve is a guide to the eye. The vertical dashed line indicates the border between the two stages in the fusion dynamics. The early stage of fusion is characterized by an average expansion velocity of $2 \mathrm{~cm} \mathrm{~s}^{-1}$. The inset displays the same data set in linear timescale. The later stage of fusion is characterized by slow opening of the fusion neck with velocity of $2.4 \mu \mathrm{m} \mathrm{s}^{-1}$ (which is the slope of the solid line in the inset).

occurs in the bilayer. Essentially, the driving forces here are the same as those responsible for the relaxation dynamics of nonporated vesicles (as charactarized by $\tau_{1}$ ). Thus, the characteristic time for this early stage of fusion, $\tau_{\text {early }}$ can be expressed as $\tau_{\text {early }} \sim \eta_{\mathrm{d}} / \sigma$, where $\eta_{\mathrm{d}}$ is the surface-dilatational viscosity of the bilayer as before, $\eta_{\mathrm{d}} \approx 3.5 \times 10^{-4} \mathrm{dyn} \mathrm{s} \mathrm{cm}^{-1}$. The membrane tension $\sigma$ should be close to the tension of rupture, i.e. of the order of $5 \mathrm{dyn}^{\mathrm{cm}^{-1}}$. Thus, for $\tau_{\text {early }}$ one obtains $100 \mu \mathrm{s}$, which is in agreement with the experimental observations for the time needed to complete the early stage of fusion.

Linear extrapolation of the data in the early stage predicts that the formation of the fusion neck with a diameter of about $10 \mathrm{~nm}$ should occur within a time period of about $250 \mathrm{~ns}$, see ref. 15 for details. It is quite remarkable that this timescale of the order of $200 \mathrm{~ns}$ was also obtained from computer simulations of a $28 \mathrm{~nm}$ vesicle fusing with a $50 \mathrm{~nm}$ tense membrane segment. ${ }^{82}$

In the later stage of fusion, the neck-expansion velocity slows down by two orders of magnitude. Here, the dynamics is mainly governed by the displacement of the volume $\Delta V$ of fluid around the fusion neck between the fused vesicles. The restoring force is related to the bending elasticity of the lipid bilayer. The parameters that determine this late relaxation are those that govern the relaxation of porated vesicles with excess area, as characterized by $\tau_{3}$, see Table 1 (note that after the two vesicles fuse, the resulting vesicle has a relatively small reduced volume and, thus, some excess area). The corresponding decay time in this later stage can be presented as $\tau_{\text {late }} \sim \eta \Delta V / \kappa$, where $\eta$ is the bulk viscosity of the media as before, $\Delta V \sim R^{3}$, and $\kappa$ is the bending elasticity modulus of the membrane. Thus, for a typical vesicle size of $R=20 \mu \mathrm{m}$, we obtain $\tau_{\text {late }} \sim 100 \mathrm{~s}$, which is the timescale that we measure for complete opening of the fusion neck.

The phenomenon of electrofusion is of particular interest, because of its vast use in cell biology and biotechnology, see ref. 2, 3 and 83 and studies cited therein. The application of 

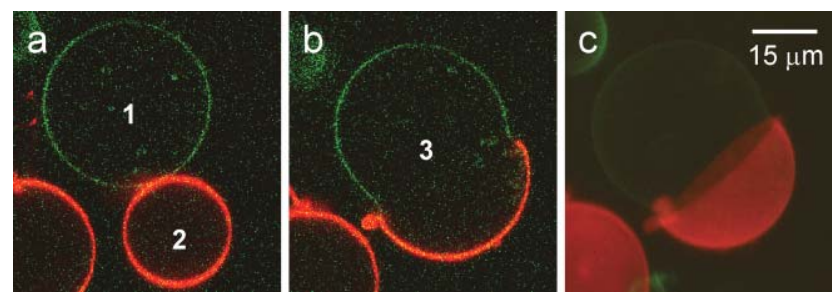

Fig. 11 Creating a multidomain vesicle by electrofusion of two vesicles of different composition as observed with fluorescence microscopy. The images $(\mathrm{a}, \mathrm{b})$ are acquired with confocal microscopy with scans nearly at the equatorial plane of the fusing vesicles. (a) Vesicle 1 is made of sphingomyelin and cholesterol (7:3) and labeled with perylene (green). Vesicle 2 is composed of dioleoyl phosphatidylcholine and cholesterol $(8: 2)$ and labeled with DiI-C18 (red). (b) The two vesicles were subjected to an electric pulse (2.2 $\mathrm{kV} \mathrm{cm}^{-1}$, duration $300 \mu \mathrm{s}$ ) and fused to form vesicle 3. (c) A three-dimensional image projection of vesicle 3 with the two domains formed from vesicles 1 and 2 .

electrofusion to cells can lead to the creation of multinucleated viable cells with new properties (this phenomenon is also known as hybridization), see e.g. ref. 3. In addition, electroporation and -fusion is often used to introduce molecules like proteins, foreign genes (plasmids), antibodies, drugs, etc. into cells.

Out of the biological context, vesicle fusion can be employed to scale-down the interaction volumes of chemical reactions and reduce it to a few picolitres or less. Thus, fusion of two vesicles of different content is an illustration for the realization of a tiny microreactor. ${ }^{84,85}$ On the other hand, fusing two vesicles, differing not in their internal content but in the composition of their membranes, provides a promising tool for studying raft-like domains in membranes. ${ }^{86-90}$ Thus, vesicle electrofusion is a very attractive experimental approach for producing multicomponent vesicles of welldefined composition.

One example for the fusion of two vesicles of different composition is given in Fig. 11. To distinguish the vesicles according to their composition, different fluorescent markers have been used. In this particular example, one of the vesicles (green) is composed of sphingomyelin and cholesterol in $7: 3$ molar ratio. The other vesicle is composed of dioleoyl phosphatidylcholine and cholesterol in $8: 2$ molar ratio. Thus, the membrane of the fused vesicle is a three-component one. At room temperature, this mixture separates into two phases, liquid ordered (rich in sphingomyelin and cholesterol) and liquid disordered (rich in dioleoyl phosphatidylcholine), which is why the final vesicle exhibits immiscible domains. The exact composition of each of these domains is not well known. However, from the domain area and the area of the initial vesicles before fusion, one can judge whether there is redistribution of the cholesterol between the domains, and eventually calculate the actual domain composition.

\section{Conclusions}

Giant vesicles as cell-size systems provide a very useful model system for resolving the effect of electric fields on lipid membranes. They allow for direct optical microscopy observation of membrane deformations in the micrometre range. We examined the behavior of giant vesicles subjected to AC fields of various frequencies. The solution conductivity appeared to be a major factor determining the overall deformation of the vesicles. A systematic study of the vesicle behavior allowed us to construct a morphology diagram of vesicle shapes as a function of field frequency and conductivity conditions. It remains to be seen whether similar behavior is found for cells. In many cases, the cell deformation will be mainly determined by the cytoskeleton flexibility rather than the membrane stiffness. However, for cells like leukocytes, it would be interesting to explore the influence of solution conductivity and field frequency on the cell shape.

Until recently, the temporal limit of optical microscopy observations with analogue video technology was in the range of milliseconds. Using fast digital imaging revealed the dynamics of electrodeformation and electroporation of vesicles subjected to DC pulses, as well as electrofusion events with microsecond resolution. Due to this high temporal resolution, new shape deformations, such as cylindrical ones with square cross-section have been detected. The observations on vesicle fusion revealed the presence of two stages of the fusion process. The expansion of the fusion neck is extremely fast in the beginning and is characterized by rates of the order of a couple of $\mathrm{cm} \mathrm{s}^{-1}$. For membranes whose thickness is only several nanometres, such a velocity is tremendous and implies that the formation of a fusion neck with a diameter of $\sim 10 \mathrm{~nm}$ should occur within a time period of $\sim 250 \mathrm{~ns}$. The later stage of fusion is a few orders of magnitude slower and is governed mainly by hydrodynamics. Finally, electrofusion of vesicles with different composition has been demonstrated to be a feasible method for creating multidomain vesicles and provides new opportunities for studying the dynamics of domain formation and stability.

\section{Acknowledgements}

We are thankful to M. Staykova and P. Peterlin for discussions and for a critical reading of the text.

\section{References}

1 Electroporation and electrofusion in cell biology, ed. E. Neumann, A. E. Sowers and C. A. Jordan, Plenum, New York, 1989.

2 Guide to electroporation and electrofusion, ed. D. C. Chang, B. M. Chassey, J. A. Saunders and A. E. Sowers, Academic Press, New York, 1992.

3 Electromanipulation of cells, ed. U. Zimmermann and G. A. Neil, CRC Press, Boca Raton, 1996.

4 A. W. Friend, Jr, E. D. Finch and H. P. Schwan, Science, 1975, 187, 357.

5 H. Engelhardt and E. Sackman, Biophys. J., 1988, 54, 495.

6 H. Engelhardt, H. Gaub and E. Sackman, Nature, 1984, 307, 378.

7 R. Heller, R. Gilbert and M. J. Jaroszeski, Adv. Drug Delivery Rev., 1999, 35, 119.

8 A. Gothelf, L. M. Mir and J. Gehl, Cancer Treat. Rev., 2003, 29, 371.

9 R. Nuccitelli, U. Pliquett, X. Chen, W. Ford, R. J. Swanson, S. J. Beebe, J. F. Kolb and K. H. Schoenbach, Biochem. Biophys. Res. Commun., 2006, 343, 351.

10 M. Golzio, J. Teissié and M. P. Rols, Proc. Natl. Acad. Sci. U. S. A., 2002, 99, 1292.

11 M. Golzio, M. P. Rols and J. Teissié, Methods, 2004, 33, 126. 
12 R. Dimova, S. Aranda, N. Bezlyepkina, V. Nikolov, K. A. Riske and R. Lipowsky, J. Phys.: Condens. Matter, 2006, 18, S1151.

13 K. A. Riske and R. Dimova, Biophys. J., 2005, 88, 1143.

14 K. A. Riske and R. Dimova, Biophys. J., 2006, 91, 1778.

15 C. K. Haluska, K. A. Riske, V. Marchi-Artzner, J.-M. Lehn, R. Lipowsky and R. Dimova, Proc. Natl. Acad. Sci. U. S. A., 2006, 103, 15841

16 K. A. Riske, N. Bezlyepkina, R. Lipowsky and R. Dimova, Biophys. Rev. Lett., 2006, 4, 387

17 K. Kinosita, Jr, I. Ashikawa, N. Saita, H. Yoshimura, H. Itoh, K. Nagayama and A. Ikegami, Biophys. J., 1988, 53, 1015.

18 D. Needham and R. M. Hochmuth, Biophys. J., 1989, 55, 1001.

19 Phospholipids handbook, ed. G. Cevc, Marcel Dekker, Inc., New York, 1993.

20 J.-Z. Bao, C. C. Davis and R. E. Schmuklert, Biophys. J., 1992, 61, 1427.

21 M. Hibino, M. Shigemori, H. Itoh, K. Nagayama and K. Kinosita, Jr, Biophys. J., 1991, 59, 209.

22 I. G. Abidor, V. B. Arakelyan, L. V. Chernomordik, Y. A. Chizmadzhev, V. F. Pastushenko and M. R. Tarasevich, J. Electroanal. Chem., 1979, 104, 37.

23 S. A. Simon and T. J. McIntosh, Methods Enzymol., 1986, 127, 511.

24 K. Olbrich, W. Rawicz, D. Needham and E. Evans, Biophys. J., 2000, 79, 321.

25 H. Aranda-Espinoza, H. Bermudez, F. S. Bates and D. E. Discher, Phys. Rev. Lett., 2001, 20, 208301.

26 R. Dimova, U. Seifert, B. Pouligny, S. Förster and H.-G. Döbereiner, Eur. Phys. J. B, 2002, 7, 241 .

27 J. Akinlaja and F. Sachs, Biophys. J., 1998, 75, 247.

28 T. Y. Tsong, Biophys. J., 1991, 60, 297.

29 J. C. Weaver and Y. A. Chizmadzhev, Bioelectrochem. Bioenerg., 1996, 41, 135.

30 W. Harbich and W. Helfrich, Z. Naturforsch., 1979, 34, 1063.

31 W. Helfrich, Z. Naturforsh., 1974, 29c, 182.

32 M. Kummrow and W. Helfrich, Phys. Rev. A: At., Mol., Opt. Phys., 1991, 44, 8356.

33 G. Niggemann, M. Kummrow and W. Helfrich, J. Phys. II, 1995, 5, 413.

34 W. Helfrich and R. M. Servuss, Nuovo Cimento Soc. Ital Fis., D, 1984, 3, 137

35 E. Evans and W. Rawicz, Phys. Rev. Lett., 1997, 79, 2379.

36 M. Mitov, P. Méléard, M. Winterhalter, M. I. Angelova and P. Bothorel, Phys. Rev. E: Stat. Phys., Plasmas, Fluids, Relat. Interdiscip. Top., 1993, 48, 628.

37 P. Méléard, C. Gerbeaud, P. Bardusco, N. Jeandaine, M. D. Mitov and L. Fernandez-Puente, Biochimie, 1998, 80, 401.

38 P. Peterlin, S. Svetina and B. Žekś, Pfluegers Arch., 2000, 439, R139.

39 H. Hyuga, K. Kinosita, Jr and N. Wakabayashi, Jpn. J. Appl. Phys., 1991, 30, 2649.

40 H. Hyuga, K. Kinosita, Jr and N. Wakabayashi, Bioelectrochem. Bioenerg., 1993, 32, 15.

41 U. Zimmermann, Biochim. Biophys. Acta, 1982, 694, 227.

42 J. L. Griffin, Exp. Cell Res., 1970, 61, 113

43 F. J. Iglesias, M. C. Lopes, C. Santamaria and A. Dominguez, Biophys. J., 1985, 48, 712.

44 V. L. Sukhorukov, H. Mussauer and U. Zimmermann, J. Membr. Biol., 1998, 163, 235.

45 E. Neumann, S. Kakorin and K. Toensing, Faraday Discuss., 1998, $111,111$.

46 S. Aranda, K. A. Riske, R. Lipowsky and R. Dimova, in preparation.

47 P. Peterlin, S. Svetina and B. Žekś, J. Phys.: Condens. Matter, 2007, 19, 136220.

48 H. Hyuga, K. Kinosita, Jr. and N. Wakabayashi, Jpn. J. Appl. Phys., 1991, 30, 1141

49 H. Hyuga, K. Kinosita, Jr. and N. Wakabayashi, Jpn. J. Appl. Phys., 1991, 30, 1333.

50 A. Sokirko, V. Pastushenko, S. Svetina and B. Žekś, Bioelectrochem. Bioenerg., 1994, 34, 101.
51 T. Griese, S. Kakorin and E. Neumann, Phys. Chem. Chem. Phys., 2002, 4, 1217.

52 S. Kakorin and E. Neumann, Colloids Surf., A, 2002, 209, 147.

53 J. Teissie and T. Y. Tsong, Biochemistry, 1981, 20, 1548.

54 R. W. Glaser, S. L. Leikin, L. V. Chernomordik, V. F. Pastushenko and A. I. Sokirko, Biochim. Biophys. Acta, 1988, 940, 275.

55 K. Kinosita, Jr, M. Hibino, H. Itoh, M. ShigemoriK. Hirano, Y. Kirino and T. Hayakawa, in Guide to Electroporation and Electrofusion, ed. D. C. Chang, B. M. Chassey, J. A. Saunders, and A. E. Sowers, Academic Press, New York, 1992.

56 D. V. Zhelev and D. Needham, Biochim. Biophys. Acta, 1993, 1147, 89.

57 O. Sandre, L. Moreaux and F. Brochard-Wyat, Proc. Natl. Acad. Sci. U. S. A., 1999, 96, 10591.

58 E. Tekle, R. D. Astumian, W. A. Friauf and P. B. Chock, Biophys. J., 2001, 81, 960.

59 N. Rodriguez, S. Cribier and N. Pincet, Phys. Rev. E: Stat. Phys, Plasmas, Fluids, Relat. Interdiscip. Top., 2006, 74, 061902.

60 R. Dimova, C. Dietrich, A. Hadjiisky, K. Danov and B. Pouligny, Eur. Phys. J. B, 1999, 12, 589

61 R. Dimova, B. Pouligny and C. Dietrich, Biophys. J., 2000, 79, 340.

62 G. Orädd, G. Wikander, G. Lindblom and L. B.-Å. Johansson, J. Chem. Soc., Faraday Trans., 1994, 90, 305.

63 F. Brochard-Wyart, P. G. de Gennes and O. Sandre, Physica A, 2000, 278, 32

64 D. C. Chang and T. S. Reese, Biophys. J., 1990, 58, 1.

65 D. S. Dimitrov and A. E. Sowers, Biochim. Biophys. Acta, 1990, 1022, 381

66 E. Tekle, R. D. Astumian and P. B. Chock, Proc. Natl. Acad. Sci. U. S. A., 1994, 91, 11512.

67 J. D. Moroz and P. Nelson, Biophys. J., 1997, 72, 2211.

68 H. Isambert, Phys. Rev. Lett., 1998, 80, 3404

69 K. C. Smith, J. C. Neu and W. Krassowska, Biophys. J., 2004, 86, 2813.

70 W. Krassowska and P. D. Filev, Biophys. J., 2007, 92, 404.

71 M. Mutz and H. Helfrich, J. Phys. France, 1990, 51, 991.

72 M. B. Schneider, J. T. Jenkins and W. W. Web, Biophys. J., 1984, 45, 891.

73 P. Sens and H. Isambert, Phys. Rev. Lett., 2002, 88, 128102.

74 Phospholipids bilayers, ed. G. Cevc and D. Marsh, John Wiley \& Sons., New York, 1987.

75 R. Lisin, B. Z. Ginzburg, M. Schlesinger and Y. Feldman, Biochim. Biophys. Acta, 1996, 1280, 34.

76 J. F. Nagle and S. Tristram-Nagle, Biochim. Biophys. Acta, 2000, $1469,159$.

77 G. Boheim, W. Hanke and H. Eibl, Proc. Natl. Acad. Sci. U. S. A., 1980, 77, 3403.

78 U. Zimmermann, Rev. Physiol. Biochem. Pharmacol., 1986, 105, 176.

79 R. Llinas, I. Z. Steinberg and K. Walton, Biophys. J., 1981, 33, 323.

80 M. Lindau and G. A. de Toledo, Biochim. Biophys. Acta, 2003, 1641, 167.

81 I. Hafes, K. Kisler, K. Berberian, G. Dernick, V. Valero, M. G. Yong, H. G. Craighead and M. Lindau, Proc. Natl. Acad. Sci. U.S. A., 2005, 102, 13879.

82 J. C. Shillcock and R. Lipowsky, Nat. Mater., 2005, 4, 225.

83 K. Kinosita, Jr. and T. Y. Tsong, Biochim. Biophys. Acta, 1977, 471, 227.

84 V. Noireaux and A. Libchaber, Proc. Natl. Acad. Sci. U. S. A., 2004, 101, 17669.

85 A. Fischer, A. Franco and T. Oberholzer, ChemBioChem, 2002, 3 , 409.

86 R. Lipowsky and R. Dimova, J. Phys.: Condens. Matter, 2003, 15, S31.

87 C. Dietrich, L. A. Bagatolli, Z. N. Volovyk, N. L. Thompson, M. Levi, K. Jacobson and E. Gratton, Biophys. J., 2001, 80, 1417.

88 T. Baumgart, S. T. Hess and W. W. Webb, Nature, 2003, 425, 821.

89 S. L. Veatch and S. L. Keller, Biophys. J., 2003, 85, 3074.

90 N. Kahya, D. Scherfeld, K. Bacia, B. Poolman and P. Schwille, J. Cell Biol., 2003, 278, 28109. 